\title{
Accelerated Partial Breast Irradiation: A New Standard of Care?
}

\author{
Tobias Forster $^{\mathrm{a}-\mathrm{c}}$ Clara Victoria Katharina Köhler ${ }^{\mathrm{a}} \quad$ Jürgen Debus ${ }^{\mathrm{a}-\mathrm{f}}$ \\ Juliane Hörner-Rieber ${ }^{a-d}$

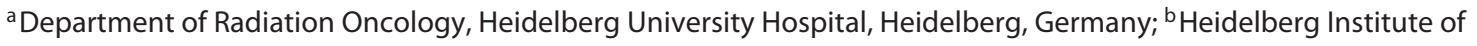 \\ Radiation Oncology (HIRO), Heidelberg, Germany; ${ }^{c}$ National Center for Tumor Diseases (NCT), Heidelberg, Germany; \\ ${ }^{\mathrm{d} C l i n i c a l}$ Cooperation Unit Radiation Oncology, German Cancer Research Center (DKFZ), Heidelberg, Germany; \\ ${ }^{e}$ Heidelberg lon-Beam Therapy Center (HIT), Department of Radiation Oncology, Heidelberg University Hospital, \\ Heidelberg, Germany; ${ }^{f}$ German Cancer Consortium (DKTK), partner site Heidelberg, Heidelberg, Germany
}

\section{Keywords}

Breast cancer · Accelerated partial breast irradiation (APBI) . Interstitial brachytherapy · External beam-based APBI . Intraoperative radiotherapy $\cdot$ MR-guided radiotherapy

\begin{abstract}
Background: Breast-conserving therapy including lumpectomy and adjuvant whole breast irradiation (WBI) has become the standard therapy for early-stage breast cancer (EBC). Without $\mathrm{WBI}$, the recurrence rate is significantly increased. However, when selecting patients at a low a priori risk of local recurrence only a small breast-cancer-specific mortality benefit, but no overall survival improvement, was detected for WBI. As most recurrences occur close to the lumpectomy cavity, accelerated partial breast irradiation (APBI) delivered exclusively to a limited volume of tissue around the initial lumpectomy site, has gained increased attention and is now discussed as an alternative to WBI for selected EBC patients. Summary: Numerous techniques for APBI (interstitial brachytherapy, external beam-based APBI, intraoperative radiotherapy, MR-guided radiotherapy) allow treatment delivery in a shorter period of time, and radiation oncologists expect to further reduce side effects by using these new techniques, with improvements in cosmetics and quality of life. In this review, we aim to describe the existing
\end{abstract}

evidence for the feasibility and effectiveness of different APBI techniques used in modern radiotherapy. Key Messages: APBI has provided outcomes similar to WBI combined with potentially reduced toxicity. While appropriate patient selection persists to be crucial for acceptable recurrence rates, the precise definition of patients suitable for APBI remains a matter of discussion. As long-term data are often still lacking, special attention should be paid to late side effects and long-term outcomes. Decision-making on appropriate treatment techniques should take into account not only local control rates, but also the impact on the patient's quality of life.

() 2020 S. Karger AG, Basel

\section{Introduction}

Over the past 30 years, breast-conserving therapy (BCT) including lumpectomy and adjuvant whole breast irradiation (WBI) has become the standard therapy for early-stage breast cancer (EBC) [1-4]. Numerous phase III randomized studies demonstrated equivalent overall survival and local control rates after BCT when compared to radical mastectomy alone [3-8]. For WBI, conventional doses vary between 40 and $50 \mathrm{~Gy}$, applied in 15-33 daily fractions for several weeks. Depending on patient 
and tumor characteristics an additional boost dose of 10 $16 \mathrm{~Gy}$ is delivered to the tumor bed [9]. Today, hypofractionated regimes with only $15-16$ fractions are the preferred regime $[10,11]$. For WBI, the entire ipsilateral breast is standardly irradiated by opposed beams oriented tangentially to the chest wall. The Early Breast Cancer Trialists' Collaborative Group (EBCTCG) demonstrated that in EBC patients adjuvant WBI reduces the 10-year risk of any first recurrence from $35.0 \%$ to $19.3 \%$ and reduces the 15-year risk of breast cancer death from 25.2 to $21.4 \%$ when compared to surgery alone [12]. In nodenegative breast cancer patients, the impact of radiotherapy on breast cancer recurrence is demonstrated by a reduction of the 10-year recurrence rate from 31.0 to $15.6 \%$ and of the 15-year breast-cancer-related mortality from 20.5 to $17.2 \%$ [12].

Nevertheless, when selecting patients at a low a priori risk of local recurrence (age $\geq 70$ years, small and estrogen-receptor-positive tumors, tamoxifen administration) only a small breast-cancer-specific mortality benefit but no overall survival improvement was reported by the EBCTCG [12]. Consequently, several trials tested the hypothesis that the addition of adjuvant WBI to endocrine therapy would not improve the outcome of patients with early-stage, low-risk breast cancer [13-18]. However, all of the individual trials identified a significant improvement for adjuvant WBI in terms of local control while no survival benefit was shown [13-18]: The BASO II study randomized patients with $\mathrm{EBC}$ in either breast-conserving surgery (BCS) followed by WBI plus tamoxifen versus only BCS plus tamoxifen versus BCS followed by WBI or $\mathrm{BCS}$ alone. Inclusion criteria were age $\leq 70$ years, nodenegative low-grade breast cancer with no lymphovascular invasion, and small tumor size $\leq 20 \mathrm{~mm}$. After a median follow-up of 10.1 years, the ipsilateral recurrence rate was more than three times higher with $10.2 \%$ for patients without WBI (BCS or BCS + tamoxifen) versus $3.0 \%$ for patients who received WBI $(\mathrm{BCS}+\mathrm{WBI}$ or $\mathrm{BCS}+\mathrm{WBI}+$ tamoxifen), but no significant improvement of overall survival was detected [13]. For the NSABP-B21 trial, EBC patients were randomized to receive $\mathrm{WBI}$ and tamoxifen versus WBI alone versus tamoxifen alone following BCS and axillary dissection. Inclusion criteria were tumor size $\leq 1 \mathrm{~cm}$, T1a-T1b, node-negative patients and estrogenreceptor-positive tumors. At an observation time of 8 years, local recurrence rates were 2.8 versus 9.3 versus $16.5 \%$ in the three treatment groups, respectively. Once again, without WBI there was an increase in the hazard rate of ipsilateral breast tumor recurrence (IBTR) by $81 \%$ compared to tamoxifen alone, but no difference in overall survival was observed [18]. In another randomized trial of Hughes et al. [15] including EBC patients aged $\geq 70$ years with small estrogen-receptor-positive tumors, a remarkable improvement in local recurrence rate was de- tected when WBI was added to tamoxifen (2\% with tamoxifen + WBI vs. $10 \%$ with tamoxifen alone), while no significant difference was seen in overall survival, distant disease-free survival, or breast preservation after a median follow-up of 12.6 years. In a meta-analysis of Matuschek et al. [19], 5 randomized trials were evaluated, including in total 3,766 mostly elderly patients with EBC treated with either adjuvant endocrine therapy or endocrine therapy plus additional WBI after BCS. They demonstrated that adjuvant hormone therapy alone resulted in significantly shorter time to local relapse compared to radiation therapy combined with hormone therapy (hazard ratio, HR: 6.8, 95\% CI: $4.23-10.93, p<0.0001)$. Therefore, even for a low-risk subset of patients omitting radiation should be no alternative.

On the one hand, postoperative irradiation of the breast is crucial for reducing local recurrence, but on the other hand, in patients with EBC most local relapses occur in the proximity of the primary tumor bed, with less than $5 \%$ in-breast recurrences elsewhere. Consequently, the main benefit of adjuvant radiation is considered to arise from the treatment delivered around the surgical cavity [20-23]. For this reason, accelerated partial breast irradiation (APBI), applied exclusively to the breast tissue surrounding the initial lumpectomy site, has gained increased attention over the past years and is now considered as an alternative to WBI for selected EBC patients [4, $24,25]$. APBI offers a way to reduce the volume of the irradiated tissue and simultaneously shortens the treatment time by hypofractionation and acceleration of radiotherapy [26]. Furthermore, APBI is more convenient for patients and is expected to be associated with less toxicity. Following WBI, acute and long-term side effects, with acute toxicity grade 2 or greater including fatigue $(18 \%)$, radiation dermatitis (40\%), and pain $(4 \%)$, are known [27]. The long course of radiotherapy during WBI is not only inconvenient for the patient, but also comes with significant social and economic impairments [27], as well as higher costs for the healthcare providers [28].

There are numerous techniques for APBI including interstitial brachytherapy, external beam-based APBI, intraoperative radiotherapy (IORT) during BCS, and new approaches using MR-guided radiotherapy (MRgRT). The commonly accepted fractionation schemes may include $34 \mathrm{~Gy}$ in 10 fractions for brachytherapy, 38 Gy in 10 fractions with external beam-based APBI, or 20-21 Gy in one single fraction with IORT $[29,30]$. While early randomized studies of the 1980s demonstrated higher local relapse rates in patients treated with APBI compared to WBI [31, 32], more recent clinical trials using improved radiotherapy techniques published diverging outcomes concerning local recurrence rates with similar outcomes in overall survival for APBI and WBI [33-36]. The longest follow-up in the literature after APBI exists for 


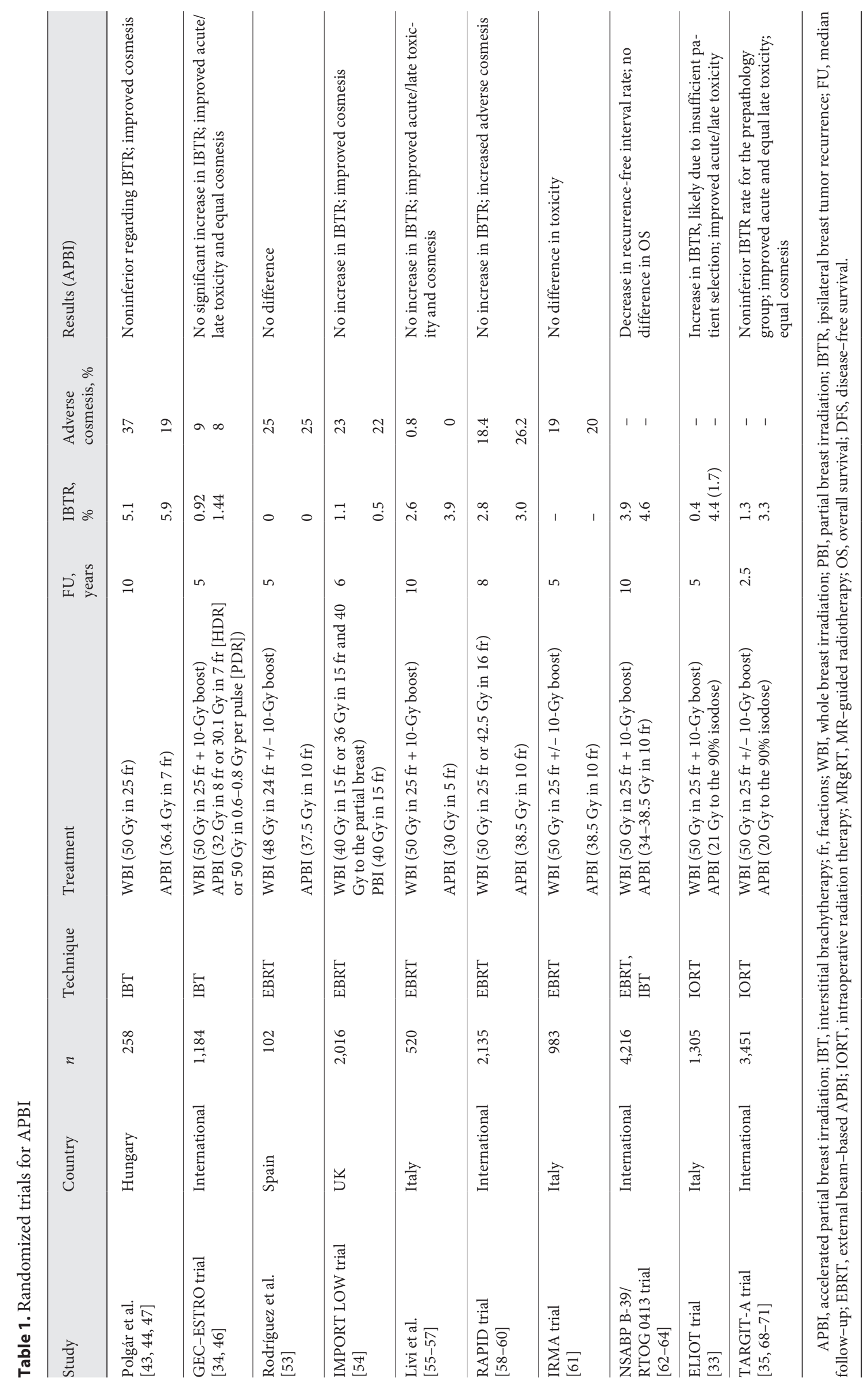


brachytherapy techniques [29]. In the field of re-irradiation for early-stage ipsilateral breast cancer recurrence (T1 N0 M0, Her2 negative, positive hormone receptor status), partial breast irradiation (PBI) using different techniques has been known to demonstrate promising local control rates for patients refusing mastectomy in favor of a second BCT for a long time [37-41].

This review aims to describe the existing evidence for advantages of APBI as well as for the feasibility and effectiveness of different APBI techniques used in modern radiotherapy. An overview of the existing randomized trials evaluating different APBI techniques is shown in Table 1.

\section{Interstitial Brachytherapy}

The most reliable data for APBI exists for brachytherapy techniques, which can be used in an intraoperative or postoperative setting [42]. In any setting, interstitial brachytherapy is predominantly dependent on the experience and skills of the treating physician, and consequently is only available in experienced institutions. Nevertheless, several remarkable trials with interstitial brachytherapy have been published so far:

A Hungarian prospective trial of Polgár et al. [43, 44] randomized 258 patients with EBC (T1 N0-1 mi, grade $1-2$, nonlobular breast cancer) after BCS to receive either WBI $(n=130)$ with 50 Gy in 25 fractions without any boost to the tumor bed or APBI $(n=128)$ delivered twice per day with HDR brachytherapy (36.4 Gy in 7 fractions). After 5 years, the actuarial rate of local relapse was noninferior with $3.4 \%$ for WBI and $4.7 \%$ for APBI as well as after 10 years with $5.1 \%$ for WBI and $5.9 \%$ for APBI ( $p=$ 0.50 and 0.77 , respectively). With respect to cosmetics, the results of Polgár et al. $[43,44]$ favored APBI with a rate of excellent to good cosmetic results of $81 \%$ for APBI versus $63 \%$ for WBI $(p<0.01)$. In a randomized trial by Lövey et al. [45], at 4 years, there was no significant difference in symptomatic fat necrosis between both treatment arms (8.5\% for WBI vs. $11.4 \%$ for APBI).

The prospective GEC-ESTRO trial randomized 1,184 patients with EBC (stage 0-IA) after BCS to receive WBI ( $n=551,50$ Gy in 25 fractions) followed by a $10-$ Gy boost to the lumpectomy cavity versus APBI $(n=633)$ delivered with HDR brachytherapy (32 Gy in 8 fractions or 30.1 Gy in 7 fractions) versus APBI applied with PDR brachytherapy (50 Gy in 0.6-0.8 Gy per pulse). The evaluation of early side effects again favored APBI for skin toxicity with grade 3 radiation dermatitis seen in $7 \%$ of cases for WBI and in only $0.2 \%$ for APBI $(p<0.0001)$. Grade $1-2$ acute toxicity occurred in $86 \%$ of patients for WBI and in only $21 \%$ for APBI $(p<0.0001)$. While breast pain was the same in both of the radiation techniques, mild hematoma and mild breast infection rates were slightly increased in APBI compared to WBI with 20 versus $2 \%$ and 5 versus $2 \%$, respectively [46]. The 5-year local recurrence rate was $0.92 \%$ (95\% CI: $0.12-1.73$ ) for WBI and $1.44 \%$ (95\% CI: $0.52-$ 2.38) for APBI $(p=0.42)$ [34]. Concerning late toxicity after a median observation time of 6.6 years, 5 -year cumulative incidence of late skin toxicity and 5-year cumulative risk for subcutaneous late effects $\geq$ grade 2 were $10.7 \%$ (95\% CI: 8.0-13.4) for WBI versus 6.9\% (95\% CI: 4.8-9.0) for APBI $(p=0.020)$ and $9.7 \%$ (95\% CI: 7.1-12.3) for WBI versus $12.0 \%$ (95\% CI: 9.4-14.7) for APBI ( $p=0.28$ ), respectively. After a follow-up of 6.6 years, breast pain increased in WBI with $11.9 \%$ compared to $8.4 \%$ for APBI $(p=0.074)$, while fair to poor cosmetic outcome was more or less the same with $9 \%$ of cases in WBI and $8 \%$ in APBI (patient rated, 10 vs. $7 \%$ when physician rated) [47].

The results of the abovementioned randomized trials are similar to findings of previously published single-arm studies using interstitial brachytherapy. Throughout these studies, local recurrence rates are slightly higher and vary from 5.2 to $15 \%$ after a follow-up of more than 10 years with excellent cosmetic outcomes [48-52].

\section{External Beam-Based APBI}

While specialized radiation oncologists are crucial for the application of brachytherapy, external beam-based APBI is widely available. However, as higher skin doses have to be expected for external beam-based APBI, important concerns regarding skin toxicity and cosmetic outcome have been raised. Nevertheless, multiple randomized trials using this radiation technique have been published, demonstrating promising results:

In a prospective trial of Rodríguez et al. [53] from Barcelona, 102 patients with $\mathrm{EBC}$ were randomized after BCS to receive either WBI ( $n=51,48$ Gy in 24 fractions with or without additional $10 \mathrm{~Gy}$ to the tumor bed) or APBI ( $n=51,37.5 \mathrm{~Gy}$ in 10 fractions delivered twice daily). After a median follow-up time of 5 years, no local recurrences were observed and no significant differences in survival rates were found. APBI reduced acute side effects and radiation doses to healthy tissues compared with WBI $(p<0.01)$. Late skin toxicity was no worse than grade 2 in either group, without significant differences between the two groups. Physician assessment showed that $>75 \%$ of patients in the APBI arm, as well as in the WBI arm, had excellent or good cosmesis, and these outcomes appear to be stable over time.

The IMPORT LOW trial, a multicenter phase III noninferiority study, randomized 2,016 patients with low-risk breast cancer (age $\geq 50$ years, unifocal invasive ductal adenocarcinoma, grade $1-3$, tumor size $\leq 3 \mathrm{~cm}, \mathrm{~T} 1-2$, N0-1) after BCS to receive one of the following: WBI $(n=674$, 40 Gy in 15 fractions, control group), WBI with a reduced 
dose of 36 Gy in 15 fractions and 40 Gy to the partial breast ( $n=673$, reduced-dose group), or partial breast irradiation ( $n=669$, PBI group) with $40 \mathrm{~Gy}$ in 15 fractions. The primary endpoint was ipsilateral local relapse, and the trial was powered to assess noninferiority of the cumulative incidence of local relapse for each of the experimental groups compared with the control group. For WBI a 2.5\% incidence of local relapse at 5 years was assumed and the IMPORT LOW trial aimed to demonstrate that an increase of more than $2.5 \%$ in the cumulative incidence of local relapse would not occur in either experimental group. After a median follow-up of 72.2 months, the 5 -year local recurrence rate was 1.1\% (95\% CI: 0.5-2.3) for WBI, $0.2 \%$ (95\% CI: 0.02-1.2) for reduced-dose WBI, and 0.5\% (95\% CI: 0.2-1.4) for PBI only. Thus, noninferiority could be claimed for both reduced-dose and partial breast radiotherapy $(p=0.003$ for the reduced-dose group and $p=0.016$ for the partial breast group, compared with the whole breast radiotherapy group). Concerning late skin toxicity, the IMPORT LOW trial favored PBI for two criteria of cosmesis: breast appearance and breast firmness achieved significantly lower rates of adverse effects ( $p=$ 0.007 and $p<0.0001$, respectively) [54].

In a randomized phase III trial of Livi et al. [55] from Florence, 520 patients with EBC (women aged $>40$ years, tumor size $<25 \mathrm{~mm}$ ) were randomly assigned to receive either conventional WBI $(n=260,50$ Gy in 25 fractions and a $10-\mathrm{Gy}$ boost to the tumor bed in 5 fractions) or APBI ( $n=260,30$ Gy in 5 fractions, intramuscular radiotherapy) after BCS. With $1.5 \%$, the local recurrence rate was equal in both arms after a median observation time of 5 years $(p=0.86)$. Acute and late toxicity ( $p=0.0001$ and $p=0.004$, respectively), as well as the cosmetic outcome $(p=0.045)$, were significantly superior for APBI compared with WBI [56]. All of the 260 patients in the APBI arm were reported to show good to excellent cosmesis at a median of 60 months of follow-up. Furthermore, the Florence study analyzed the health-related quality of life at the end of treatment as well as after 2 years of follow-up using the EORTC QLQ-C30 and BR-23 questionnaires. Already at the end of treatment, global health status $(p=0.0001)$ and most scores relative to the functional and symptoms scales of the QLQ-C30 questionnaire favored APBI. Functional and symptom scales of the BR-23 questionnaire were also superior for APBI, including body perception, future perspective, and breast and arm symptoms. Results were confirmed after 2 years of follow-up with QLQ-C30 global health status $(p=0.0001)$ as well as most functional and symptoms scales being still in favor of APBI. Body perception, future perspective, and breast and arm symptoms also remained superior in the APBI group, as assessed by the BR-23 questionnaire [55].
More recently, an update for the 10-year results has been presented at San Antonio Breast Cancer Symposium 2019. At 10 years, $3.9 \%$ of patients treated with APBI had experienced ipsilateral breast cancer recurrence compared with $2.6 \%$ in those who received WBI (HR: 1.57, 95\% CI: $0.56-4.41, p=0.39$ ), which was not determined to be statistically significant. Furthermore, no significant differences were reported for locoregional recurrence rates with 3.9 versus $3.0 \%$ as well as for overall survival rates with 92.7 and $93.7 \%$ for the APBI and the WBI arms, respectively [57].

In a phase III trial, the Canadian RAPID study, 2,135 patients with $\mathrm{EBC}$ (age $\geq 40$ years, invasive or noninvasive breast cancer, tumor size $\leq 3 \mathrm{~cm}$ ) were randomized to receive either WBI ( $n=1065,50$ Gy in 25 fractions or 42.5 Gy in 16 fractions) or APBI ( $n=1070,38.5$ Gy in 10 fractions given twice daily) after BCS. The primary outcome was IBTR, analyzed by intention to treat. The RAPID trial was designed as a noninferiority trial on the basis of an expected 5-year IBTR rate of $1.5 \%$ in the WBI group. Noninferiority was assumed if a 1.5\% increase in the IBTR rate could be excluded for the APBI group. After a median follow-up of 8.6 years, the 8 -year cumulative rates of IBTR were $3.0 \%$ (95\% CI: $1.9-4.0)$ in the APBI group and $2.8 \%$ (95\% CI: 1.8-3.9) in the WBI group. Hence, APBI was demonstrated to be noninferior [58]. While acute toxicity (grade $>2$, within 3 months) occurred less frequently in the APBI group ( $28 \%$ of patients) than in the WBI group ( $45 \%$ of patients, $p<0.0001$ ), late toxicity (grade $>2$, later than 3 months) was more common in patients treated with APBI (32 vs. 13\%, $p<0.0001$ ) [58]. Using a hypofractionated and highly accelerated form of APBI, the RAPID study demonstrated unexpected poor late cosmetic outcome for the APBI arm [59]. After 3, 5, and 7 years of follow-up, adverse cosmesis remained more frequent in the APBI group, with an absolute difference at 3 years of 11.3\% (95\% CI: 7.5-15.0), 5 years: $16.5 \%$ (95\% CI: $12.5-$ 20.4), and 7 years: $17.7 \%$ (95\% CI: 12.9-22.3) [58]. The authors concluded that a 6 -h interval between fractions may not suffice for normal tissue repair, resulting in increased grade 1-2 toxicities in the APBI arm [59]. Peterson et al. [60] reported the predictors of poor cosmesis of the RAPID study. While clinical and pathologic characteristics were similar in both treatment arms, tumor location (worse cosmesis associated with outer location), smoking, higher age (per 10 years), and larger seroma volume (per $10 \mathrm{~cm}^{2}$ ) were found to be significant predictors of adverse cosmetic outcome $(p<0.05)$.

For the IRMA trial, a prospective multicenter study from Italy, 983 patients with EBC (age $\geq 49$ years, tumor size $<3 \mathrm{~cm}$, pN0-1) were randomly assigned to receive WBI (50 Gy in 25 daily fractions with or without a 10-Gy boost to the lumpectomy cavity) or APBI (38.5 Gy in 10 fractions delivered twice daily) after BCS. The results were 
presented at the European Society of Radiation Oncology (ESTRO) meeting in 2017. Although similar fractionation schemes were applied as in the RAPID trial, no difference was observed in adverse cosmesis between both treatment arms after a median follow-up of 5 years (20\% for APBI vs. $19 \%$ for WBI after 3 years when physician rated and 14\% for APBI as well as WBI after 3 years when patient rated). Acute skin toxicity grade 3 was rare in both treatment schemes $(0 \%$ in APBI vs. $1.4 \%$ in WBI) and no further acute toxicity grade $\geq 3$ was observed. Late skin toxicity and late subcutaneous tissue toxicity grade $3-4$ was 0.42 and $1.5 \%$ for APBI versus 0.79 and $1.2 \%$ for WBI ( $p=0.4$ and $p=0.7)$, respectively. The IRMA trial is still awaiting results for local control rates and overall survival [61].

Results of the NSABP B-39/RTOG 0413 trial, a prospective phase III equivalence study, have recently been presented at the San Antonia Breast Cancer Symposium 2019 [62] and have now been published in Lancet [63]. The NSABP B-39/RTOG 0413 trial randomized 4,216 patients with EBC (stage 0, I or II, $0-3$ positive axillary nodes) to receive WBI ( $n=2109,50$ Gy in 25 fractions with a sequential boost to the surgical cavity) or APBI ( $n=2107,34-38.5$ Gy in 10 fractions given twice daily with either brachytherapy or external beam radiation) after BCS. The primary endpoint for analysis was IBTR (invasive and noninvasive) as first recurrence, analyzed in the intention-to-treat population, applying an equivalency test on the basis of a 50\% margin increase in the HR (90\% CI for the observed HR between 0.667 and 1.5 for equivalence). At a median follow-up of 10.2 years, 90 (4\%) of 2,089 women eligible for the primary outcome in the APBI group and $71(3 \%)$ of 2,036 women in the WBI group had an IBTR (HR: 1.22, 90\% CI: 0.94-1.58), resulting in a 10-year cumulative IBTR incidence of 4.6\% (95\% CI: 3.7-5.7) in the APBI group versus 3.9\% (95\% CI: 3.1$5.0)$ in the WBI group [63]. To declare APBI and WBI equivalent in respect to the risk of IBTR, the $90 \%$ CI for the observed HR comparing APBI to WBI would have had to be entirely between 0.667 and 1.5 . However, an HR of 1.22 with a $90 \%$ CI of $0.94-1.58$ was observed, which did not meet the equivalence criteria and hence the trial favored WBI. No statistically significant differences existed between APBI and WBI in distant disease-free interval (time from randomization to first diagnosis of distant recurrence) $(p=0.15)$, overall survival $(p=0.35)$, or disease-free survival $(p=0.11)$ [63]. While grade 2 toxicity was slightly decreased in the APBI group (44 vs. 59\% for WBI, not significant), grade 1 and 3 toxicity were slightly higher with $40 \%$ for APBI versus $31 \%$ for WBI and $9.6 \%$ for APBI versus $7.1 \%$ for WBI, respectively (not significant). Grade $4-5$ toxicity was found to be 0.5 versus $0.3 \%$, respectively [63]. Nevertheless, the absolute difference in the 10-year rate of IBTR was reassuringly low at $<1 \%$ (4.8\% APBI vs. $4.1 \% \mathrm{WBI}$ ) [62-64].
The SHARE trial, another large randomized phase III study closed to accrual in 2017. Results from this trial are still pending. The SHARE trial randomized 1,006 patients with EBC (age $\geq 50$ years, tumor size $<2 \mathrm{~cm}$, all invasive) to receive either standard fractionated or hypofractionated WBI or APBI with external beam radiation (38.5 Gy delivered over 10 twice-daily fractions) $[65,66]$.

\section{Intraoperative Radiotherapy}

From a patient perspective, the most comfortable technique for APBI may be IORT delivered in a single fraction during BCS. IORT may not only improve patient compliance, but also decrease irradiation of healthy organs, reduce the dose to the skin, and induce lower treatment costs [67]. Several large clinical trials for IORT have been published so far:

In the ELIOT trial, a prospective randomized study, highly energized electrons were used to deliver radiotherapy to the lumpectomy cavity during surgery. A total of 1,305 patients with EBC (age $\geq 48$ years, tumor size $\leq 2.5$ $\mathrm{cm}$ ) were randomly assigned to receive either a single dose of $21 \mathrm{~Gy}$ intraoperatively prescribed to the $90 \%$ isodose given to the tumor bed $(n=651)$, or WBI $(n=654,50$ Gy in 25 fractions with a 10-Gy boost to the lumpectomy cavity). The rate for IBTR was significantly increased in patients treated with IORT compared to WBI (4.4 vs. $0.4 \%$, $p=0.0001$ ) after 5 years. However, for a subgroup of lowrisk patients (tumor size $<2 \mathrm{~cm}$, positive nodes $<3$, grade $1-2$, estrogen receptor positive, not triple negative), the rate for IBTR was $1.7 \%$ at 5 years. With regard to skin toxicity, patients in the IORT arm presented with significantly lower rates of skin erythema, dryness, hyperpigmentation, and pruritus compared to patients in the WBI arm. Moreover, the pulmonary fibrosis rate was higher in the WBI group, while an increased rate of fat necrosis occurred in the IORT group (17 vs. 7\% for WBI, $p=0.04$ ) [33].

Another IORT study, the prospective TARGIT-A trial, randomized 3,451 patients with EBC (age $\geq 45$ years, tumor size $\leq 3.5 \mathrm{~cm}$, unifocal invasive ductal carcinoma) to receive a single shot IORT of $20 \mathrm{~Gy}$ to the resection cavity $(n=1,721$, application of a $1.5-$ to $5.0-\mathrm{cm}$ spherical applicator, $50 \mathrm{kV}$ energy X-rays) versus standard WBI ( $n=1730,50$ Gy in 25 fractions with or without a 10-Gy boost to the tumor bed). IORT was either delivered during BCS (prepathology arm) or after BCS in a second surgical intervention (postpathology arm). However, due to risk factors seen during surgery or on final pathology (tumor-free margin $<1 \mathrm{~mm}$, extensive in situ component, or unexpected invasive lobular carcinoma), $21 \%$ of patients in the prepathology IORT arm received an additional WBI according to institutional standard ranging from 40 to $56 \mathrm{~Gy}$. Additional WBI was scheduled as per protocol 
(risk-adapted management). At a median follow-up of 2 years and 5 months, the risk for ipsilateral recurrence was $3.3 \%$ in the IORT arm compared to $1.3 \%$, in the WBI arm $(p=0.042)$. The noninferiority margin of the trial $(2.5 \%)$ was met by the prepathology group: $2.1 \%(1.1-4.2)$ for IORT versus $1.1 \%(0.5-2.5)$ for WBI $(p=0.31)$, but not by the postpathology group: $5.4 \%(3.0-9.7)$ for IORT versus $1.7 \%(0.6-4.9)$ for WBI $(p=0.069)$. For skin toxicities, a decreased rate of grade 3-4 adverse events was reported for IORT versus WBI ( 0.2 vs. $0.8 \%, p=0.029)$. Interestingly, there were less non-breast-cancer-related deaths in the IORT arm ( 1.4 vs. $3.5 \%$ in the WBI arm, $p=0.0086$ ). The authors ascribed these findings to supposed higher rates of cardiac events and second malignancies in the WBI group [35]. Except for telangiectasia, which occurred solely in WBI patients (17\%), late side effects were more or less the same in both treatment arms, including fibrosis, breast edema, retraction, lymphedema, and pain. Breast and arm symptoms were decreased in the IORT arm [68]. After a median follow-up of 2 years and 5 months, results of cosmesis were equal between the treatment groups, while breast-related quality of life was increased for IORT compared with WBI $[35,69,70]$.

Recently, results from the TARGIT-E trial were presented at ASTRO 2019. The TARGIT-E trial, a single-arm IORT study, enrolled 526 elderly patients with low-risk breast cancer ( $\geq 70$ years, cT1 $1 / 2(<3.5 \mathrm{~cm}), \mathrm{cN} 0, \mathrm{cM} 0$, invasive carcinoma of no special type) to receive a single dose of 20 Gy (IORT, low-energy X-rays) during BCS. In the case of risk factors present in the final pathology (larger size, other histology, free margin $<1 \mathrm{~cm}$, lymphatic vessel invasion, positive nodes, multifocal/central lesions, extensive intraductal component), patients were treated with WBI (46-50 Gy, 1.8-2.0 Gy per fraction). The primary outcome was the local recurrence rate measured at $2.5,5$, and 7.5 years. Of 526 patients, 474 were included in the analysis: 347 (73\%) received IORT only, $99(21 \%)$ IORT plus whole breast radiotherapy, 22 (5\%) whole breast radiotherapy only, and 7 (1\%) surgery only without subsequent adjuvant therapies. After a median follow-up of 3 years (range: 2-79 months), 4 ipsilateral in-breast recurrences after 11,33, 42, and 43 months were reported, leading to estimated local recurrence rates of 0.2 and $1.5 \%$ at 2.5 and 5 years, respectively. Overall, 20 patients died ( 3 breast-cancer-related deaths, 17 non-breast-cancer-related deaths), resulting in estimated overall survival rates of 98.9 and $91.4 \%$ at 2.5 and 5 years, respectively [71].

Both the ELIOT and the TARGIT-A trial provide robust data supposing superiority of IORT with regard to toxicity and long-term cosmesis, which may have attributed to the reduced volume of irradiated breast tissue [30]. While in the ELIOT trial patient selection was not optimal to achieve acceptable local control rates, the results of the TARGIT-A trial were impaired due to the tim- ing of IORT. In the postpathology arm, IORT was delivered during a second operation. Consequently, another prospective randomized phase II study, the COSMOPOLITAN trial, was designed at Heidelberg University, selecting patients on the basis of the ASTRO consensus statements for APBI. For the COSMOPOLITAN trial, a total of 202 patients with selected low-risk EBC (age $>50$ years, tumor size $<2.5 \mathrm{~cm}$, grade $1-2$, estrogen-receptorpositive tumors, node negative) will be randomized to receive either single-dose IORT with electrons during BCS (21 Gy, prescribed to the $90 \%$ isodose) or postoperative hypofractionated WBI (40.05 Gy in 15 daily fractions, 5 weeks after surgery). In contrast to the ELIOT trial, patient selection criteria will be more restrictive and IORT will be compared to hypofractionated WBI, as hypofractionation has become the standard treatment following BCT for low-risk EBC patients. Recruitment of patients started in late 2019 and is expected to be completed in 2021. The primary endpoint will be patient-reported fatigue. Further endpoints evaluated are tumor control, overall survival, disease-free survival, acute and chronic toxicity, quality of life, and cosmesis. First results are expected in 2022 [72]. The flowchart of the COSMOPOLITAN trial is depicted in Figure 1.

\section{MR-Guided Radiotherapy}

One of the most recent approaches for APBI is MRgRT [73]. MRgRT enables direct visualization of the lumpectomy cavity as well as characterization and eventual tracking of anatomical motion by using real-time imaging. Especially in soft tissue, where targets need accurate definition, MR guidance may mark the beginning of a new era $[73,74]$. In comparison to brachytherapy or IORT techniques, external beam-based APBI requires additional margins to account for chest wall movement, which may result in larger irradiation volumes. MRgRT may potentially overcome this disadvantage of external beam-based radiotherapy. On the one hand, MRgRT offers a superior soft-tissue contrast for precise detection of the cavity. On the other hand, MRgRT enables advanced motion management strategies like gating, providing "real-time" anatomical feedback. Respiratory gating is a process which allows for continuous monitoring of the cavity motion during normal breathing. Radiation is only delivered when the tumor cavity is tracked in exactly the right position in the real-time 4-D cine MRI, and the treatment beam automatically turns off if the tumor cavity moves out of the target field (Fig. 2). Therefore, required safety margins and hence treated volumes can be reduced.

However, availability of next-generation hybrid MRLINAC systems is still limited, and MRgRT remains more time consuming [73]. Nevertheless, some experience in 


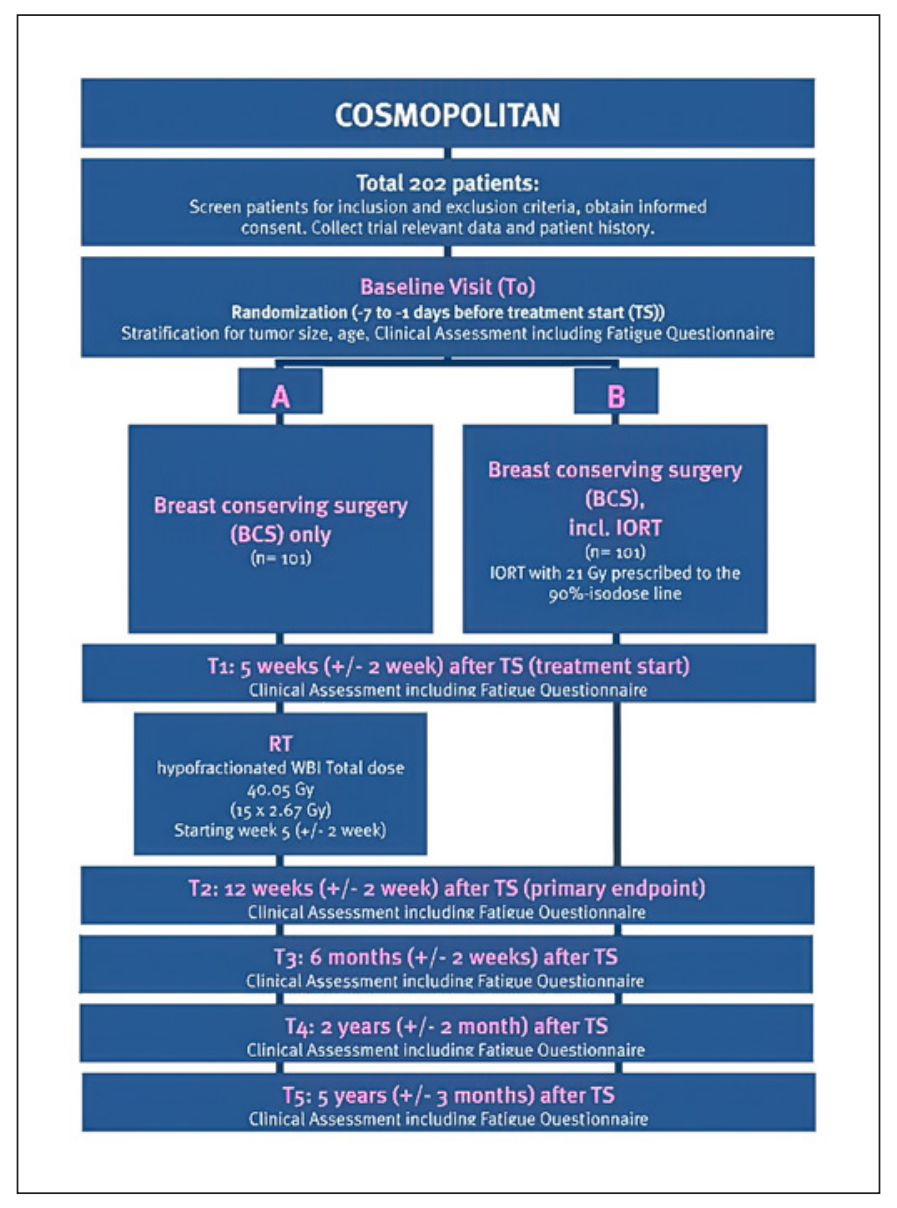

Fig. 1. Flowchart of the COSMOPOLITAN trial.

the field of MRgRT used for APBI has already been gathered. In a prospective registry evaluating APBI using a 0.35-T MRgRT system, 30 patients with EBC (stage 0-1) were enrolled after BCS. Patients were treated with APBI (38.5 Gy in 10 fractions delivered twice daily). 4-D MR real-time imaging during treatment was applied for visualizing the surgical cavity. Compared to conventionally external beam-based APBI, the treated volume could therefore be reduced by $52 \%$ when using MRgRT. Results for efficacy and cosmesis of this MR-guided APBI approach are still pending [75].

\section{Patient Selection}

For patient selection, several societies have published recommendations to define whether patients are suitable or not for APBI, taking into account risk factors regardless of the selected APBI techniques [76-78]. The European Society for Radiotherapy and Oncology (GECESTRO), as well as the American Society for Radiation Oncology (ASTRO) and the American Brachytherapy Society (ABS) recommend suitable patients to be 50 years of age or older, to have a negative node status and absence of lympho-vascular space invasion. Tumor size must be $\leq 3 \mathrm{~cm}$ based on ABS and GEC-ESTRO recommendations and $\geq 2 \mathrm{~cm}$ according to the ASTRO. While the GEC-ESTRO and ASTRO consider only invasive ductal carcinoma with positive estrogen receptor status to be suitable for APBI, the ABS regards all invasive carcinomas and ductal carcinomas in situ as suitable for PBI, irrespective of the estrogen receptor status. The GECESTRO recommendations furthermore exclude patients diagnosed with multifocal carcinoma and patients having received neoadjuvant chemotherapy. In contrast, the ASTRO and ABS do not take these criteria into consideration [76-79]. The different recommendations for APBI indicate the important criteria for appropriate patient selection. However, most data underlying these recommendations are weak and a universal consensus has not been found yet. Individual factors such as breast size, tumor bed location, and distance of the surgical cavity to the skin surface might also have to be taken into account. Consequently, current guidelines recommend the application of APBI preferably within clinical trials [10].

\section{Final Remarks}

In summary, several large randomized phase III trials showed that APBI has provided outcomes similar to WBI combined with potentially reduced toxicity $[34,43,44$, $46,47,54-56]$. However, appropriate patient selection remains crucial to achieve ipsilateral tumor recurrence rates that are noninferior to those reported after WBI. This was underlined again by the recently published results of the NSABP B-39 trial [63]. Nevertheless, the precise definition of the subset of low-risk EBC patients that are suitable for APBI remains a matter of discussion. Special attention should be paid to late side effects and longterm outcomes. As the thorough assessment of late toxicities from high-dose focal radiotherapy requires at least 5 years of follow-up and long-term data are often still lacking, more robust information will be gathered in the future after a longer observation time [29, 80]. Nowadays, reported toxicity with APBI is generally mild; nevertheless, some authors showed higher incidences of fat necrosis and skin toxicity especially when using external beambased APBI with hypofractionated and highly accelerated schedules [59]. These reports have fired concerns about cosmetic outcome following external beam-based APBI.

Many uncertainties remain concerning radiobiological aspects of APBI, as different techniques require a large heterogeneity of dose prescriptions, dose fractionation, and target volumes. Results from direct comparison of different APBI techniques (interstitial brachytherapy, external beam-based APBI, IORT, MRgRT) are still lacking. 
Fig. 2. MR-guided APBI. A, B Treatment plan and dose-volume histogram of MRguided APBI delivered at Heidelberg University with a total dose of 40.05 Gy in 15 fractions as second breast-conserving therapy due to local recurrence. The resection cavity is shown in orange, while the planning target volume is delineated in red. $\mathbf{C}-\mathbf{F}$ MR real-time cine imaging with the target (resection cavity) within the specified gating margin (C) and the beam turned on (D) and $>3 \%$ of the target located outside of the specified gating margin $(\mathbf{E})$ and the beam turned off (F).
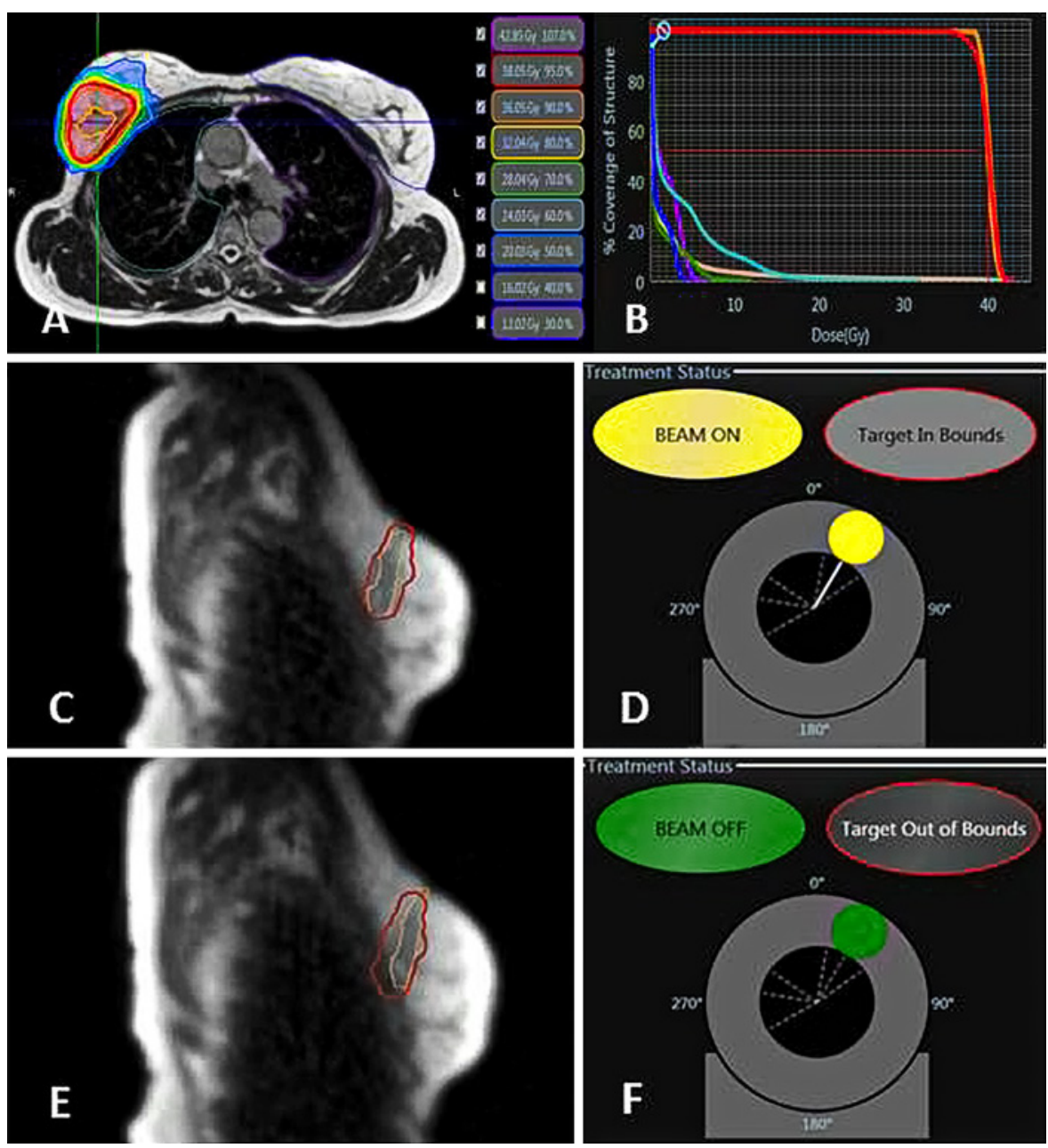

Furthermore, not all techniques are extensively available, as the required technical skills vary considerably depending on the selected APBI technique. In general, decisionmaking on appropriate treatment techniques (WBI or different APBI techniques) should take into account not only improvements in local control rates, but also treatment-associated toxicity and the impact on the patient's quality of life. The findings of ongoing trials will widen our understanding of the role of APBI in the therapy of EBC patients in the near future [72].

\section{Conclusion}

During the last years, increasingly profound data has been gathered to support APBI using various techniques as a treatment alternative to WBI for selected low-risk EBC patients. APBI is especially attractive, as it potentially offers fewer side effects combined with a reduction in treatment time, thus minimizing treatment burden for patients and healthcare systems. However, for ensuring acceptable recurrence rates, appropriate patient selection remains essential. Updated and new results from several pending trials are eagerly awaited to complement current evidence and assist clinicians and patients with shared decision-making about the use of APBI.

\section{Disclosure Statement}

The authors have no conflicts of interest to declare.

\section{Funding Sources}

The resources and facilities of the Medical Faculty of Heidelberg University were used in conducting this review. There was no additional source of funding.

\section{Author Contributions}

T.F. carried out the literature search and analysis and drafted the manuscript. J.H.-R. helped with the literature search and to draft the manuscript. C.V.K.K. helped to draft the manuscript. All authors participated in manuscript revisions and approved the final manuscript. 


\section{References}

1 Marta GN, Hanna SA, Martella E, Silva JL, Carvalho Hde A. Early stage breast cancer and radiotherapy: update. Rev Assoc Med Bras (1992). 2011 Jul-Aug;57(4):459-64.

2 Poortmans P. Evidence based radiation oncology: breast cancer. Radiother Oncol. 2007 Jul;84(1):84-101.

3 Veronesi U, Cascinelli N, Mariani L, Greco M, Saccozzi R, Luini A, et al. Twenty-year follow-up of a randomized study comparing breast-conserving surgery with radical mastectomy for early breast cancer. N Engl J Med. 2002 Oct;347(16):1227-32.

4 Fisher B, Anderson S, Bryant J, Margolese RG, Deutsch M, Fisher ER, et al. Twenty-year follow-up of a randomized trial comparing total mastectomy, lumpectomy, and lumpectomy plus irradiation for the treatment of invasive breast cancer. N Engl J Med. 2002 Oct; 347(16):1233-41

5 van Dongen JA, Bartelink H, Fentiman IS, Lerut $\mathrm{T}$, Mignolet F, Olthuis $\mathrm{G}$, et al. Randomized clinical trial to assess the value of breastconserving therapy in stage I and II breast cancer, EORTC 10801 trial. J Natl Cancer Inst Monogr. 1992;(11):15-8.

6 Jacobson JA, Danforth DN, Cowan KH, d'Angelo T, Steinberg SM, Pierce L, et al. Tenyear results of a comparison of conservation with mastectomy in the treatment of stage I and II breast cancer. N Engl J Med. 1995 Apr; 332(14):907-11.

7 Blichert-Toft M, Rose C, Andersen JA, Overgaard M, Axelsson CK, Andersen KW, et al.; Danish Breast Cancer Cooperative Group. Danish randomized trial comparing breast conservation therapy with mastectomy: six years of life-table analysis. J Natl Cancer Inst Monogr. 1992;(11):19-25.

8 van Maaren MC, de Munck L, de Bock GH, Jobsen JJ, van Dalen T, Linn SC, et al. 10 year survival after breast-conserving surgery plus radiotherapy compared with mastectomy in early breast cancer in the Netherlands: a population-based study. Lancet Oncol. 2016 Aug; 17(8):1158-70.

9 Bartelink H, Maingon P, Poortmans P, Weltens C, Fourquet A, Jager J, et al.; European Organisation for Research and Treatment of Cancer Radiation Oncology and Breast Cancer Groups. Whole-breast irradiation with or without a boost for patients treated with breast-conserving surgery for early breast cancer: 20-year follow-up of a randomised phase 3 trial. Lancet Oncol. 2015 Jan;16(1):47-56.

10 Deutsche Krebsgesellschaft DK. A., S3-Leitlinie Früherkennung, Diagnostik, Therapie und Nachsorge des Mammakarzinoms. Leitlinienprogramm Onkologie. Available from: http://www.leitlinienprogramm-onkologie. de/leitlinien/mammakarzinom/.

11 Miranda FA, Teixeira LA, Heinzen RN, de Andrade FE, Hijal T, Buchholz TA, et al. Accelerated partial breast irradiation: current status with a focus on clinical practice. Breast J. 2019 Jan;25(1):124-8.

12 Darby S, McGale P, Correa C, Taylor C, Arriagada $\mathrm{R}$, Clarke $\mathrm{M}$, et al.; Early Breast Cancer Trialists' Collaborative Group (EBCTCG). Effect of radiotherapy after breast-conserving surgery on 10-year recurrence and 15-year breast cancer death: meta-analysis of individual patient data for 10,801 women in 17 randomised trials. Lancet. 2011 Nov;378(9804): 1707-16.

13 Blamey RW, Bates T, Chetty U, Duffy SW, Ellis IO, George D, et al. Radiotherapy or tamoxifen after conserving surgery for breast cancers of excellent prognosis: British Association of Surgical Oncology (BASO) II trial. Eur J Cancer. 2013 Jul;49(10):2294-302.

14 Fyles AW, McCready DR, Manchul LA, Trudeau ME, Merante P, Pintilie M, et al. Tamoxifen with or without breast irradiation in women 50 years of age or older with early breast cancer. N Engl J Med. 2004 Sep; 351(10):963-70.

15 Hughes KS, Schnaper LA, Bellon JR, Cirrincione CT, Berry DA, McCormick B, et al. Lumpectomy plus tamoxifen with or without irradiation in women age 70 years or older with early breast cancer: long-term follow-up of CALGB 9343. J Clin Oncol. 2013 Jul; 31(19):2382-7.

16 Kunkler IH, Williams LJ, Jack WJ, Cameron DA, Dixon JM; PRIME II investigators. Breast-conserving surgery with or without irradiation in women aged 65 years or older with early breast cancer (PRIME II): a randomised controlled trial. Lancet Oncol. 2015 Mar;16(3):266-73.

17 Pötter R, Gnant M, Kwasny W, Tausch C, Handl-Zeller L, Pakisch B, et al.; Austrian Breast and Colorectal Cancer Study Group. Lumpectomy plus tamoxifen or anastrozole with or without whole breast irradiation in women with favorable early breast cancer. Int J Radiat Oncol Biol Phys. 2007 Jun;68(2):334-40.

18 Fisher B, Bryant J, Dignam JJ, Wickerham DL, Mamounas EP, Fisher ER, et al.; National Surgical Adjuvant Breast and Bowel Project. Tamoxifen, radiation therapy, or both for prevention of ipsilateral breast tumor recurrence after lumpectomy in women with invasive breast cancers of one centimeter or less. J Clin Oncol. 2002 Oct;20(20):4141-9.

19 Matuschek C, Bölke E, Haussmann J, Mohrmann S, Nestle-Krämling C, Gerber PA, et al. The benefit of adjuvant radiotherapy after breast conserving surgery in older patients with low risk breast cancer- a meta-analysis of randomized trials. Radiat Oncol. 2017 Mar; 12(1):60.

20 Boyages J, Recht A, Connolly JL, Schnitt SJ, Gelman R, Kooy H, et al. Early breast cancer: predictors of breast recurrence for patients treated with conservative surgery and radiation therapy. Radiother Oncol. 1990 Sep; 19(1):29-41

21 Kurtz JM, Amalric R, Brandone H, Ayme Y, Jacquemier J, Pietra JC, et al. Local recurrence after breast-conserving surgery and radiotherapy. Frequency, time course, and prognosis. Cancer. 1989 May;63(10):1912-7.

22 Veronesi U, Marubini E, Mariani L, Galimberti V, Luini A, Veronesi P, et al. Radiotherapy after breast-conserving surgery in small breast carcinoma: long-term results of a randomized trial. Ann Oncol. 2001 Jul;12(7): 997-1003.
23 Liljegren G, Holmberg L, Adami HO, Westman G, Graffman S, Bergh J; Uppsala-Orebro Breast Cancer Study Group. Sector resection with or without postoperative radiotherapy for stage I breast cancer: five-year results of a randomized trial. J Natl Cancer Inst. 1994 May;86(9):717-22.

24 Mannino M, Yarnold JR. Local relapse rates are falling after breast conserving surgery and systemic therapy for early breast cancer: can radiotherapy ever be safely withheld? Radiother Oncol. 2009 Jan;90(1):14-22.

25 Salvadori B, Marubini E, Miceli R, Conti AR, Cusumano F, Andreola S, et al. Reoperation for locally recurrent breast cancer in patients previously treated with conservative surgery. Br J Surg. 1999 Jan;86(1):84-7.

26 Vicini FA, Baglan KL, Kestin LL, Mitchell C, Chen PY, Frazier RC, et al. Accelerated treatment of breast cancer. J Clin Oncol. 2001 Apr; 19(7):1993-2001.

27 Whelan TJ, Olivotto IA, Parulekar WR, Ackerman I, Chua BH, Nabid A, et al.; MA.20 Study Investigators. Regional Nodal Irradiation in Early-Stage Breast Cancer. N Engl J Med. 2015 Jul;373(4):307-16.

28 Darby SC, Ewertz M, McGale P, Bennet AM, Blom-Goldman U, Brønnum D, et al. Risk of ischemic heart disease in women after radiotherapy for breast cancer. N Engl J Med. 2013 Mar;368(11):987-98.

29 Bennion NR, Baine M, Granatowicz A, Wahl AO. Accelerated partial breast radiotherapy: a review of the literature and future directions. Gland Surg. 2018 Dec;7(6):596-610.

30 Dutta SW, Showalter SL, Showalter TN, Libby B, Trifiletti DM. Intraoperative radiation therapy for breast cancer patients: current perspectives. Breast Cancer (Dove Med Press). 2017 Apr;9:257-63.

31 Dodwell DJ, Dyker K, Brown J, Hawkins K, Cohen D, Stead M, et al. A randomised study of whole-breast vs tumour-bed irradiation after local excision and axillary dissection for early breast cancer. Clin Oncol (R Coll Radiol). 2005 Dec;17(8):618-22.

32 Ribeiro GG, Magee B, Swindell R, Harris M, Banerjee SS. The Christie Hospital breast conservation trial: an update at 8 years from inception. Clin Oncol (R Coll Radiol). 1993; 5(5):278-83.

33 Veronesi U, Orecchia R, Maisonneuve P, Viale G, Rotmensz N, Sangalli C, et al. Intraoperative radiotherapy versus external radiotherapy for early breast cancer (ELIOT): a randomised controlled equivalence trial. Lancet Oncol. 2013 Dec;14(13):1269-77.

34 Strnad V, Ott OJ, Hildebrandt G, KauerDorner D, Knauerhase H, Major T, et al.; Groupe Européen de Curiethérapie of European Society for Radiotherapy and Oncology (GEC-ESTRO). 5-year results of accelerated partial breast irradiation using sole interstitial multicatheter brachytherapy versus whole-breast irradiation with boost after breast-conserving surgery for low-risk invasive and in-situ carcinoma of the female breast: a randomised, phase 3, non-inferiority trial. Lancet. 2016 Jan;387(10015):22938. 
35 Vaidya JS, Wenz F, Bulsara M, Tobias JS, Joseph DJ, Keshtgar M, et al.; TARGIT trialists' group. Risk-adapted targeted intraoperative radiotherapy versus whole-breast radiotherapy for breast cancer: 5-year results for local control and overall survival from the TARGIT-A randomised trial. Lancet. 2014 Feb; 383(9917):603-13.

36 Marta GN, Macedo CR, Carvalho HA, Hanna SA, da Silva JL, Riera R. Accelerated partial irradiation for breast cancer: systematic review and meta-analysis of 8653 women in eight randomized trials. Radiother Oncol. 2015 Jan;114(1):42-9.

37 Forster T, Akbaba S, Schmitt D, Krug D, El Shafie R, Oelmann-Avendano J, et al. Second breast conserving therapy after ipsilateral breast tumor recurrence - a 10-year experience of re-irradiation. J Contemp Brachytherapy. 2019 Aug;11(4):312-9.

38 Hannoun-Levi JM, Resch A, Gal J, KauerDorner D, Strnad V, Niehoff P, et al.; GECESTRO Breast Cancer Working Group. Accelerated partial breast irradiation with interstitial brachytherapy as second conservative treatment for ipsilateral breast tumour recurrence: multicentric study of the GEC-ESTRO Breast Cancer Working Group. Radiother Oncol. 2013 Aug;108(2):226-31.

39 Resch A, Fellner C, Mock U, Handl-Zeller L, Biber E, Seitz W, et al. Locally recurrent breast cancer: pulse dose rate brachytherapy for repeat irradiation following lumpectomy- a second chance to preserve the breast. Radiology. 2002 Dec;225(3):713-8.

40 Arthur DW, Winter KA, Kuerer HM, Haffty B, Cuttino L, Todor DA, et al. Effectiveness of Breast-Conserving Surgery and 3-Dimensional Conformal Partial Breast Reirradiation for Recurrence of Breast Cancer in the Ipsilateral Breast: The NRG Oncology/RTOG 1014 Phase 2 Clinical Trial. JAMA Oncol. 2019 Nov.

41 Boehm KM, Chen J, Trichter S, Abdallah H, Zhuo R, Nowak EJ, et al. Characterization of Recurrences Following Second Breast-Conserving Surgery with Intraoperative Radiotherapy. Ann Surg Oncol. 2019 Oct. https:// doi.org/10.1245/s10434-019-07908-5.

42 Strnad V, Major T, Polgar C, Lotter M, Guinot JL, Gutierrez-Miguelez C, et al. ESTROACROP guideline: Interstitial multi-catheter breast brachytherapy as Accelerated Partial Breast Irradiation alone or as boost - GECESTRO Breast Cancer Working Group practical recommendations. Radiother Oncol. 2018 Sep;128(3):411-20.

43 Polgár C, Fodor J, Major T, Németh G, Lövey $\mathrm{K}$, Orosz Z, et al. Breast-conserving treatment with partial or whole breast irradiation for low-risk invasive breast carcinoma-5-year results of a randomized trial. Int J Radiat Oncol Biol Phys. 2007 Nov;69(3):694-702.

44 Polgár C, Fodor J, Major T, Sulyok Z, Kásler M. Breast-conserving therapy with partial or whole breast irradiation: ten-year results of the Budapest randomized trial. Radiother Oncol. 2013 Aug;108(2):197-202.

45 Lövey K, Fodor J, Major T, Szabó E, Orosz Z, Sulyok Z, et al. Fat necrosis after partial-breast irradiation with brachytherapy or electron irradiation versus standard whole-breast radio- therapy-4-year results of a randomized trial. Int J Radiat Oncol Biol Phys. 2007 Nov;69(3): 724-31.

46 Ott OJ, Strnad V, Hildebrandt G, KauerDorner D, Knauerhase H, Major T, et al.; Groupe Européen de Curiethérapie of European Society for Radiotherapy and Oncology (GEC-ESTRO). GEC-ESTRO multicenter phase 3-trial: Accelerated partial breast irradiation with interstitial multicatheter brachytherapy versus external beam whole breast irradiation: Early toxicity and patient compliance. Radiother Oncol. 2016 Jul;120(1): 119-23.

47 Polgár C, Ott OJ, Hildebrandt G, KauerDorner D, Knauerhase H, Major T, et al.; Groupe Européen de Curiethérapie of European Society for Radiotherapy and Oncology (GEC-ESTRO). Late side-effects and cosmetic results of accelerated partial breast irradiation with interstitial brachytherapy versus whole-breast irradiation after breast-conserving surgery for low-risk invasive and in-situ carcinoma of the female breast: 5 -year results of a randomised, controlled, phase 3 trial. Lancet Oncol. 2017 Feb;18(2):259-68.

48 Polgár C, Major T, Fodor J, Sulyok Z, Somogyi A, Lövey $\mathrm{K}$, et al. Accelerated partialbreast irradiation using high-dose-rate interstitial brachytherapy: 12-year update of a prospective clinical study. Radiother Oncol. 2010 Mar;94(3):274-9.

49 White J, Winter K, Kuske RR, Bolton JS, Arthur DW, Scroggins T, et al. Long-Term Cancer Outcomes From Study NRG Oncology/ RTOG 9517: A Phase 2 Study of Accelerated Partial Breast Irradiation With Multicatheter Brachytherapy After Lumpectomy for EarlyStage Breast Cancer. Int J Radiat Oncol Biol Phys. 2016 Aug;95(5):1460-5.

50 Rabinovitch R, Winter K, Kuske R, Bolton J, Arthur D, Scroggins T, et al. RTOG 95-17, a Phase II trial to evaluate brachytherapy as the sole method of radiation therapy for Stage I and II breast carcinoma-year-5 toxicity and cosmesis. Brachytherapy. 2014 Jan-Feb;13(1): 17-22.

51 Hattangadi JA, Powell SN, MacDonald SM, Mauceri T, Ancukiewicz M, Freer P, et al. Accelerated partial breast irradiation with lowdose-rate interstitial implant brachytherapy after wide local excision: 12-year outcomes from a prospective trial. Int J Radiat Oncol Biol Phys. 2012 Jul;83(3):791-800.

52 Kamrava M, Kuske RR, Anderson B, Chen P, Hayes J, Quiet C, et al. Outcomes of Breast Cancer Patients Treated with Accelerated Partial Breast Irradiation Via Multicatheter Interstitial Brachytherapy: The Pooled Registry of Multicatheter Interstitial Sites (PROMIS) Experience. Ann Surg Oncol. 2015 Dec; 22(S3 Suppl 3):S404-11.

53 Rodríguez N, Sanz X, Dengra J, Foro P, Membrive I, Reig A, et al. Five-year outcomes, cosmesis, and toxicity with 3-dimensional conformal external beam radiation therapy to deliver accelerated partial breast irradiation. Int $\mathrm{J} \mathrm{Ra}$ diat Oncol Biol Phys. 2013 Dec;87(5):1051-7.

54 Coles CE, Griffin CL, Kirby AM, Titley J, Agrawal RK, Alhasso A, et al.; IMPORT Trialists. Partial-breast radiotherapy after breast conservation surgery for patients with early breast cancer (UK IMPORT LOW trial): 5-year results from a multicentre, randomised, controlled, phase 3, non-inferiority trial. Lancet. 2017 Sep;390(10099):1048-60.

55 Livi L, Meattini I, Marrazzo L, Simontacchi G, Pallotta S, Saieva C, et al. Accelerated partial breast irradiation using intensity-modulated radiotherapy versus whole breast irradiation: 5-year survival analysis of a phase 3 randomised controlled trial. Eur J Cancer. 2015 Mar;51(4):451-63.

56 Meattini I, Saieva C, Miccinesi G, Desideri I, Francolini G, Scotti V, et al. Accelerated partial breast irradiation using intensity modulated radiotherapy versus whole breast irradiation: health-related quality of life final analysis from the Florence phase 3 trial. Eur J Cancer. 2017 May;76:17-26.

57 Meattini I. Partial breast irradiation may be as effective as whole breast irradiation in preventing recurrence in patients with early breast cancer. 2019 San Antonio Breast Cancer Symposium; 2019; San Antonio.

58 Whelan TJ, Julian JA, Berrang TS, Kim DH, Germain I, Nichol AM, et al.; RAPID Trial Investigators. External beam accelerated partial breast irradiation versus whole breast irradiation after breast conserving surgery in women with ductal carcinoma in situ and node-negative breast cancer (RAPID): a randomised controlled trial. Lancet. 2019 Dec;394(10215): 2165-72.

59 Olivotto IA, Whelan TJ, Parpia S, Kim DH, Berrang T, Truong PT, et al. Interim cosmetic and toxicity results from RAPID: a randomized trial of accelerated partial breast irradiation using three-dimensional conformal external beam radiation therapy. J Clin Oncol. 2013 Nov;31(32):4038-45.

60 Peterson D, Truong PT, Parpia S, Olivotto IA, Berrang T, Kim DH, et al.; RAPID trial investigators. Predictors of adverse cosmetic outcome in the RAPID trial: an exploratory analysis. Int J Radiat Oncol Biol Phys. 2015 Apr; 91(5):968-76

61 Meduri B, Baldissera A, Galeandro M, Donini E, Tolento G, Giacobazzi P, et al. OC-0568: Accelerated PBI VS standard radiotherapy (IRMA trial): interim cosmetic and toxicity results. Radiother Oncol. 2017;123:S303.

62 Vicini FA. Abstract GS4-04: Primary results of NSABP B-39/RTOG 0413 (NRG Oncology): A randomized phase III study of conventional whole breast irradiation (WBI) versus partial breast irradiation (PBI) for women with stage 0, I, or II breast cancer, in San Antonio Breast Cancer Symposium. 2019, AACR. https://doi.org/10.1158/1538-7445. SABCS18-GS4-04.

63 Vicini FA, Cecchini RS, White JR, Arthur DW, Julian TB, Rabinovitch RA, et al. Longterm primary results of accelerated partial breast irradiation after breast-conserving surgery for early-stage breast cancer: a randomised, phase 3, equivalence trial. Lancet. 2019 Dec;394(10215):2155-64.

64 NSABP B-39. NSABP B-39, RTOG 0413: A Randomized Phase III Study of conventional whole breast irradiation versus partial breast irradiation for women with stage 0 , I, or II breast cancer. Clin Adv Hematol Oncol. 2006 Oct;4(10):719-21. 
65 Belkacemi Y, Bourgier C, Kramar A, Auzac G, Dumas I, Lacornerie T, et al. SHARE: a French multicenter phase III trial comparing accelerated partial irradiation versus standard or hypofractionated whole breast irradiation in breast cancer patients at low risk of local recurrence. Clin Adv Hematol Oncol. 2013 Feb; 11(2):76-83.

66 Standard or hypofractionated radiotherapy versus accelerated partial breast irradiation (APBI) for breast cancer (SHARE) [cited 2020 January 14]. Available from: https://clinicaltrials.gov/ct2/show/NCT01247233.

67 Grobmyer SR, Lightsey JL, Bryant CM, Shaw C, Yeung A, Bhandare N, et al. Low-kilovoltage, single-dose intraoperative radiation therapy for breast cancer: results and impact on a multidisciplinary breast cancer program. J Am Coll Surg. 2013 Apr;216(4):617-23; discussion 623-4.

68 Sperk E, Welzel G, Keller A, Kraus-Tiefenbacher U, Gerhardt A, Sütterlin M, et al. Late radiation toxicity after intraoperative radiotherapy (IORT) for breast cancer: results from the randomized phase III trial TARGIT A. Breast Cancer Res Treat. 2012 Aug;135(1): 253-60.

69 Welzel G, Boch A, Sperk E, Hofmann F, Kraus-Tiefenbacher U, Gerhardt A, et al. Radiation-related quality of life parameters after targeted intraoperative radiotherapy versus whole breast radiotherapy in patients with breast cancer: results from the randomized phase III trial TARGIT-A. Radiat Oncol. 2013 Jan;8(1):9.
70 Corica T, Nowak AK, Saunders CM, Bulsara M, Taylor M, Vaidya JS, et al. Cosmesis and breast-related quality of life outcomes after intraoperative radiation therapy for early breast cancer: a substudy of the TARGIT-A Trial. Int J Radiat Oncol Biol Phys. 2016 Sep; 96(1):55-64.

71 Sperk E. First per protocol analysis of the Prospective Phase II Study of Intraoperative Radiotherapy (IORT) in Elderly Patients with Small Breast Cancer: Targit E(lderly). Int J Radiat Oncol Biol Phys. 2019 Sep;105(1 Suppl):S8. Available from: https://www.redjournal.org/article/S0360-3016(19)31228-3/ fulltext.

72 ClinicalTrials.gov. Intraoperative electron radiotherapy for low-risk early breast cancer (COSMOPOLITAN). 2019. Available from: https://clinicaltrials.gov/ct2/show/study/ NCT03838419.

73 Corradini S, Alongi F, Andratschke N, Belka C, Boldrini L, Cellini F, et al. MR-guidance in clinical reality: current treatment challenges and future perspectives. Radiat Oncol. 2019 Jun;14(1):92.

74 Nachbar M, Mönnich D, Boeke S, Gani C, Weidner N, Heinrich V, et al. Partial breast irradiation with the $1.5 \mathrm{~T}$ MR-Linac: first patient treatment and analysis of electron return and stream effects. Radiother Oncol. 2019 Dec;145:30-5.

75 Acharya S, Fischer-Valuck BW, Mazur TR, Curcuru A, Sona K, Kashani R, et al. Magnetic resonance image guided radiation therapy for external beam accelerated partial-breast irradiation: evaluation of delivered dose and intrafractional cavity motion. Int J Radiat Oncol Biol Phys. 2016 Nov;96(4):785-92.
76 Hepel JT, Arthur D, Shaitelman S, Polgár C, Todor D, Zoberi I, et al. American Brachytherapy Society consensus report for accelerated partial breast irradiation using interstitial multicatheter brachytherapy. Brachytherapy. 2017 Sep - Oct;16(5):919-28.

77 Polgár C, Van Limbergen E, Pötter R, Kovács G, Polo A, Lyczek J, et al.; GEC-ESTRO breast cancer working group. Patient selection for accelerated partial-breast irradiation (APBI) after breast-conserving surgery: recommendations of the Groupe Européen de Curiethérapie-European Society for Therapeutic Radiology and Oncology (GEC-ESTRO) breast cancer working group based on clinical evidence (2009). Radiother Oncol. 2010 Mar; 94(3):264-73.

78 Correa C, Harris EE, Leonardi MC, Smith BD, Taghian AG, Thompson AM, et al. Accelerated Partial Breast Irradiation: Executive summary for the update of an ASTRO Evidence-Based Consensus Statement. Pract Radiat Oncol. 2017 Mar - Apr;7(2):73-9.

79 Smith BD, Arthur DW, Buchholz TA, Haffty BG, Hahn CA, Hardenbergh PH, et al. Accelerated partial breast irradiation consensus statement from the American Society for Radiation Oncology (ASTRO). Int J Radiat Oncol Biol Phys. 2009 Jul;74(4):987-1001.

80 Franco P, Iorio GC, Bartoncini S, Airoldi M, De Sanctis C, Castellano I, et al. De-escalation of breast radiotherapy after conserving surgery in low-risk early breast cancer patients. Med Oncol. 2018 Apr;35(5):62. 